\title{
Dentro y fuera de las tumbas campaniformes en Camino de las Yeseras: ¿una segunda vida para los muertos? ${ }^{1}$ Inside and outside the Beaker tombs of Camino de las Yeseras: A second life for the deads?
}

\author{
Corina Liesau ${ }^{1}$ \\ Patricia Ríos ${ }^{2}$ \\ Concepción Blasco ${ }^{2}$ \\ Irene Ortiz ${ }^{2}$
}

\section{Resumen}

Mientras que en los contextos funerarios los esqueletos humanos completos o parciales son bien reconocibles, no suele ocurrir lo mismo en aquellos que se encuentran de forma aislada o mezclados con artefactos y otros restos de origen animal. Su identificación, aunque sólo sea de forma fragmentaria, es una línea de estudio que no siempre ha tenido la atención que merece debido a que los análisis arqueofaunísticos suelen emprenderse de forma tardía en relación con los de otros materiales y ello impide, en la mayoría de las ocasiones, que los restos antropológicos fuera de las tumbas sean identificados y estudiados adecuadamente.

Sin embargo, precisamente algunos de los hallazgos aislados no reconocidos durante las tareas de campo pueden llegar a aportar una información muy valiosa para aproximarnos al mundo de la compleja simbología del Calcolítico desde una perspectiva en la que el apoyo empírico resulta fundamental. El presente trabajo se basa fundamentalmente en los restos antropológicos recuperados en las campañas de excavación realizadas en el yacimiento de Camino de las Yeseras desde el año 2003, la representatividad esquelética humana dentro y fuera de las tumbas campaniformes, en la que se percibe una extracción y traslado de restos seleccionados que a su vez vuelven a incorporarse a otras estructuras no funerarias, como las cabañas, hoyos y fosos. En muchos casos, se confirma una interesante asociación de mandíbulas con otros restos de perro.

Palabras clave: Centro Peninsular, Calcolítico, Campaniforme, mandíbulas humanas y de perros.

\begin{abstract}
Complete or fragmented human remains usually are well recognized at funerary contexts, but it does not happen with those isolated bones mixed with faunal remains and other materials. Their identification out of graves is a research line that not always has deserved attention due to archaeozoological analysis are frequently done after other analysis materials, and this circumstance usually prevent appropriate identification of their bones and teeth.

However, these human remains not recognized during field works can provide important information to approach us to the complex chalcolithic symbolic world where an empiric support is fundamental. The present paper is based fundamentally on a selection of these types of anthropological remains, mainly identified and selected from the faunal studies, recovered during field works at "Camino de las Yeseras" site since 2003 campaign. A brief discussion about skeletal representativity inside Bell Beaker tombs is presented, because they mainly show movements and transfers of selected bones beside their association with other faunal remains in pits, huts and ditches. In many cases in an interesting association with human mandibles and other dog remains are documented.
\end{abstract}

Keywords: Central Iberia, Chalcolithic, Bell Beaker, human and dog mandibles.

Proyecto de I+D: "Las Sociedades Calcolíticas en el interior peninsular: Origen y desarrollo de los grandes poblados de la Prehistoria Reciente. Estudios Interdisciplinares.” REF: HAR2016-77600-P. Programa Estatal de Fomento de la Investigación Científica y Técnica de Excelencia, Subprograma Estatal de Generación del Conocimiento, MINECO, Gobierno de España.

\footnotetext{
Departamento de Prehistoria y Arqueología. Universidad Autónoma de Madrid. corina.liesau@uam.es
} 


\section{INTRODUCCIÓN}

Entre los muchos temas que ha tratado la Dra. Isabel Rubio a lo largo de su dilatada trayectoria académica no faltan aquellos dedicados al mundo simbólico en diferentes contextos y áreas geográficas (Rubio 2001-02, 2004a; 2004b, 2010-11). Por ello, hemos considerado oportuno participar en su merecido homenaje con unas breves reflexiones sobre el significado de la presencia de determinadas porciones esqueléticas humanas y animales en algunas estructuras como un posible indicio de un patrón de comportamiento de carácter simbólico.

El presente trabajo se basa fundamentalmente en los estudios realizados del material osteológico de Camino de las Yeseras desde la campaña del 2003, localizado tanto en contextos funerarios como no funerarios. Desde entonces hemos estudiado sistemáticamente numerosas estructuras -tramos de foso, cabañas, tumbas, hoyos- con una metodología y análisis de laboratorio que prestan especial atención a la recuperación de restos arqueobiológicos con técnicas muy exhaustivas (Liesau et al., 2008; Blasco et al., 2009, 2011; Gómez et al., 2011; Ríos, 2011; Liesau et al., 2014a, 2014b).

A medida que se ha ido avanzando en los estudios hemos podido identificar numerosos restos humanos de diferentes estructuras asociados a fauna que, desde el punto de vista tafonómico, ya han permitido obtener datos interesantes (Gómez et al., 2011). Teniendo en cuenta que hasta la fecha se han estudiado los materiales osteológicos de más de 500 estructuras del yacimiento, fundamentalmente hoyos y cabañas, nos permite tener una perspectiva más amplia en torno a la presencia de los restos humanos en el yacimiento. Mientras que en algunos casos, la recuperación de determinadas porciones óseas permite caracterizar algunas pautas relativas a la selección anatómica de huesos y su lugar y forma de deposición, en otras estructuras las secuencias son mucho más complejas; es el caso de los fosos y el área central, de los que también hemos ofrecido una serie de avances, estudios e interpretaciones puntuales (Vega et al., 2010; Ríos, 2011; Ríos et al., 2014; Liesau et al., 2013-2014; Chorro, 2012; Liesau, 2017). Con todo ello podemos destacar que los restos humanos fuera de las tumbas no abundan ni parecen estar tan dispersos como cabe suponer al tratarse de un lugar que presenta más de mil años de ocupación prehistórica.

A medida que avancemos en los resultados, el registro aquí presentado es susceptible de ampliarse considerablemente, pero todo apunta hacia unos hábitos de colocación de determinadas porciones esqueléticas humanas $\mathrm{y}$ de algunos animales que reflejan pautas deposicionales de un lenguaje no escrito condicionado por los espacios $\mathrm{y}$ contextos que representan.

\section{RESTOS HUMANOS EN CONTEXTOS FUNERARIOS:} ¿DESCANSO ETERNO O BÚSQUEDA DE LOS ANCESTROS?

La necesidad de abordar este tema se enmarca dentro de las investigaciones que, desde comienzo del siglo XXI, se centran en la búsqueda de una explicación a la función de los "poblados de hoyos" en general, y de los recintos de fosos en particular, para la que ya no sirve una única causa, sino la suma de actividades diversas y recurrentes de unas ocupaciones humanas que por su estacionalidad y prácticas de abandono, no generan asentamientos permanentes (Márquez y Jiménez, 2010) y donde "la abundantísima cultura material que colmata los hoyos (...) son el resultado de deposiciones intencionadas, es decir, contextos antrópicos de abandono" (Márquez y Mata, 2016: 43), entendiendo por "abandono", no el desinterés o el rechazo (basurero) sino la materialización explícita del final de un ciclo o biografía de los sujetos y objetos (Kopytoff, 1986; Marshall y Gosden, 1999; Brück, 2006; Liesau et al., 2013-14; Blanco, 2014).

Desde esta óptica es indispensable prestar especial atención a los contextos en los que se encuentran todos y cada uno de los restos arqueológicos sin excepción. Sin embargo, a pesar de que en el marco de las investigaciones cada vez son más numerosos los estudios arqueobotánicos, arqueozoológicos y antropológicos, sus resultados pocas veces se presentan contextualizados y asociados, tanto a los restos industriales a los que acompañan, como a las unidades estratigráficas en la que se localizan. Se trata de indicios fundamentales para interpretar la biografía de la estructura, incluido el momento en el que se producen los últimos procesos deposicionales o de relleno, así como del propio resto, ya que tras el óbito se producen con frecuencia traslados y manipulaciones post mortem que añaden significados complementarios a las inhumaciones primarias (Blasco et al., 2014; Liesau et al., 2014b).

Son muchas las causas que han ayudado a que estas prácticas de extracción y traslado hayan podido pasar inadvertidas. Entre ellas podemos destacar a los agentes postdeposicionales que han afectado a la conservación diferencial de los restos esqueléticos, como son las características del sedimento, los procesos disolutivos por la acción hídrica (Arteaga, 2011), las erosiones radiculares, o las fracturas por la presión que ejercen las tierras y piedras que cubren los enterramientos. La falta de formación cualificada, la ausencia de protocolos de extracción normalizados, el carácter de urgencia de algunas intervenciones y la ausencia de flotado o cribado del sedimento funerario son otros aspectos que inciden negativamente en un buen registro de los huesos conservados ya que precisamente los de menor tamaño -rótulas, cárpales, tarsales, falanges, sesamoideos, etc.- son fundamentales para la interpretación del carácter primario o secundario de las inhumaciones (Duday y Guillon, 2006).

\subsection{Traslados de restos humanos}

En el caso de los restos humanos es habitual que tras la muerte los cuerpos sean depositados en una tumba individual o colectiva sin manipulaciones posteriores a las del enterramiento, aunque tampoco es infrecuente la presencia de restos parciales a consecuencia de varios reacondi- 
cionamientos o reducciones dentro de una misma tumba e incluso por traslados desde otras tumbas (Blasco et al., 2014; Liesau et al. 2014a).

Las inhumaciones parciales no son excepcionales en el registro funerario calcolítico no campaniforme y campaniforme del yacimiento, especialmente en el caso de las tumbas colectivas o múltiples (Liesau et al, 2008:109; Blasco et al, 2009; 2011; Vega et al, 2010; Liesau et al. 2014a, Ríos et al., 2014). También son habituales los hallazgos de huesos humanos dispersos en distintos puntos del yacimiento fuera de los contextos funerarios: zona central, algún tramo de foso, o en estructuras de tipo cabaña (Gómez et al, 2011; Liesau et al., 2013-14). Y aunque estos traslados parecen un hecho probado, demostrarlo fehacientemente es algo excepcional y condicionado en gran parte al azar. Afortunadamente estos traslados en Camino de las Yeseras se han detectado en especial para para algunos individuos que se inhumaron con ajuares campaniformes, pero al no conocer el yacimiento en toda su extensión la falta de información es considerable. Además, hemos de tener en cuenta que el sector de población con campaniforme representa tan solo una veintena de individuos, frente al medio centenar no campaniformes hasta ahora documentados, hemos podido comprobar que éstas prácticas ya se realizaron en etapas anteriores.

Aunque se han documentado algunas tumbas sin ajuares campaniformes con restos parciales o con reacondicionamiento de los cuerpos en Camino de las Yeseras, estas manipulaciones son más frecuentes y complejas en aquellas que contienen recipientes campaniformes. Atendiendo a la representatividad esquelética de las diferentes tumbas campaniformes podemos destacar algunas categorías interesantes que no parecen tener paralelos con otras inhumaciones calcolíticas sin la mencionada vajilla:

1. Tumbas intactas con inhumaciones primarias: Tan sólo dos tumbas corresponden esta categoría

\begin{tabular}{|c|c|c|c|c|c|}
\hline TUMBA & TIPO & INDIVIDUO & AJUAR CERÁMICO & ADORNOS & OTROS \\
\hline $\begin{array}{c}\text { Área Funeraria } 2 \\
\text { Hipogeo } \\
\text { A35/03-III }\end{array}$ & $\begin{array}{l}\text { primaria } \\
\text { intacta }\end{array}$ & 1 adulto joven & Cazuela & $\begin{array}{l}\text { Placas y cuentas de oro, } \\
\text { botones y cuentas de marfil }\end{array}$ & Cinabrio \\
\hline $\begin{array}{c}\text { Área Funeraria } 2 \\
\text { Covacha } \\
\text { A35/03-X }\end{array}$ & $\begin{array}{l}\text { primaria } \\
\text { alterada }\end{array}$ & 1 adulto & Vaso y cuenco & - & - \\
\hline \multirow{2}{*}{$\begin{array}{c}\text { Área Funeraria } 2 \\
\text { Covacha } \\
\text { A35/03-VII }\end{array}$} & $\begin{array}{l}\text { primaria } \\
\text { intacta }\end{array}$ & 1 adulto & 2 Cuencos & - & - \\
\hline & $\begin{array}{c}\text { primaria } \\
\text { desplazada }\end{array}$ & 1 infantil & 1 Cuenco & - & - \\
\hline \multirow{3}{*}{$\begin{array}{c}\text { Área Funeraria } 1 \\
\text { Covacha } \\
\text { A31/E01-II }\end{array}$} & primaria & 1 adulto & \multirow{3}{*}{3 Cuencos } & - & - \\
\hline & secundaria & 1 senil & & - & Cinabrio \\
\hline & de criba & 1 feto/ neonato? & & - & - \\
\hline \multirow{3}{*}{$\begin{array}{c}\text { Área Funeraria } 1 \\
\text { Hipogeo } \\
\text { A31/01-I }\end{array}$} & secundario & 1 senil & \multirow{3}{*}{$\begin{array}{l}2 \text { vasos, } \\
1 \text { cazuela, } \\
1 \text { cuenco incompletos }\end{array}$} & \multirow{3}{*}{ Placa de oro } & \multirow{3}{*}{$\begin{array}{l}\text { Cinabrio y molino con } \\
\text { mano }\end{array}$} \\
\hline & secundario & 1 senil & & & \\
\hline & secundario & 1 adulto & & & \\
\hline \multirow{4}{*}{$\begin{array}{l}\text { Tumba en hoyo } \\
\text { geminado } \\
\text { A21 E06 }\end{array}$} & secundario & 1 adulto de gran talla & \multirow{4}{*}{$\begin{array}{l}\text { Fragmentos de } 15 \\
\text { recipientes completos o } \\
\text { medios ( } 5 \text { vasos, } 9 \text { cuencos } \\
\text { y } 1 \text { cazuela) }\end{array}$} & \multirow{4}{*}{$\begin{array}{l}1 \text { cuenta de oro, un botón } \\
\text { de diente de cachalote con } \\
\text { doble perforación simple y } \\
\text { un punzón de cobre }\end{array}$} & \multirow{4}{*}{$\begin{array}{l}\text { Cinabrio }+2 \text { molinos } \\
\text { enteros, } 8 \text { molinos } \\
\text { fragmentados, } 3 \text { manos de } \\
\text { molino + otros macrolíticos }\end{array}$} \\
\hline & secundario & 1 adulto & & & \\
\hline & secundario & 1 adulto & & & \\
\hline & secundario & 1 infantil & & & \\
\hline \multirow{3}{*}{$\begin{array}{c}\text { Área Funeraria } 3 \\
\text { Covacha } 1 \\
\text { F } 5\end{array}$} & primaria & 1 adulto & 1 vaso y 1 cuenco & - & - \\
\hline & secundario & 1adulto & - & - & - \\
\hline & secundario & 1 maduro & - & - & - \\
\hline \multirow{3}{*}{$\begin{array}{c}\text { Área Funeraria } 3 \\
\text { Covacha } 2 \\
\text { F } 5\end{array}$} & primario & 1 adulto & \multirow{3}{*}{1 vaso, 1 cazuela 2 cuencos } & \multirow{3}{*}{ - } & \multirow{3}{*}{$\begin{array}{l}1 \text { mortero } \\
1 \text { molino } \\
1 \text { punzón }\end{array}$} \\
\hline & secundario & 1 adulto & & & \\
\hline & secundario & 1 senil & & & \\
\hline
\end{tabular}

Tabla 1. Relación de los tipos de enterramientos campaniformes con sus ajuares documentados en Camino de las Yeseras. 
(tabla 1). Se trata de dos inhumaciones individuales, un hipogeo y una covacha insertas en la denominada Área Funeraria 2 (Liesau et al., 2008; Blasco et al., 2009; Gómez et al., 2011).

2. Tumbas intactas con al menos un esqueleto desplazado: En el Área funeraria 2 (A35/E03VII), hay un esqueleto infantil desplazado bajo el cuerpo de una mujer, en posición primaria y cuya esqueletización se produjo en un espacio colmatado (Gómez et al., 2011; Liesau et al., 2014) (tabla 1).

3. Tumbas con enterramientos primarios y secundarios: En esta categoría se incluyen tres de las tumbas campaniformes documentadas en Camino de las Yeseras (tabla 1). Se trata de tres covachas: la del Área funeraria 1 (A31/E01-II) y las dos covachas del Área Funeraria 3 (cabaña 5). En todas ellas se depositó una inhumación principal acompañada de una o de varias secundarias. Éstas se reducen a las porciones más representativas.

Parece que, en los tres casos, tras la última inhumación, correspondiente a la que permanecía en posición primaria, se realizó el sellado de las cámaras mediante grandes losas o nódulos de sílex y grandes cantos con el fin de salvaguardar los restos humanos y ajuares que contenían, objetivo que se consiguió pese a la posterior ocupación del yacimiento durante el II milenio AC e incluso en época romana. De momento no hemos podido verificar el tiempo que estas tumbas pudieron haber sido accesibles con un cierre provisional, quizás leñoso, para efectuar las sucesivas inhumaciones con los correspondientes reacondicionamientos y reducciones de los restos previos a los sucesivos enterramientos.

4. Tumbas alteradas: En esta categoría se pueden incluir tumbas que presentan una extracción de restos antropológicos y ajuares funerarios: El hipogeo del área funeraria 1 y una tumba en hoyo del área funeraria 21 (tabla 1). Su desmantelado debió de producirse tras la exhumación parcial de los restos humanos y sus ajuares en el hoyo y un nicho que presentaba. En ambos casos se hallaban selladas, aunque al interior quedaban solo restos óseos sin conexión anatómica y fragmentos cerámicos muy dispersos, seguramente como resultado de enterramientos diferidos a lo largo de un lapso temporal que no podemos precisar, pero siguiendo los criterios de Teresa Andrés (Andrés, 1998: 157), se trata de acumulaciones sucesivas a lo largo de siglos de uso, tal y como atestiguan las dataciones de C14 del hoyo del área 21 (Ríos, 2013).

\section{RESTOS HUMANOS EN CONTEXTOS NO FUNERARIOS}

Como ya se ha comentado, a medida que los trabajos de campo y de laboratorio avanzan, se identifican más restos humanos fuera de los contextos funerarios como consecuencia de actos intencionados, los cuales aportan un nuevo significado de carácter no menos relevante que los propiamente fúnebres. Algunos de ellos han sido recuperados de hoyos, fosos, cabañas domésticas o de carácter colectivo (fig. 1 y 2), aunque en estas circunstancias y contextos no es posible saber si tales restos corresponden a individuos inhumados con ajuares campaniformes o no, o si previamente fueron expuestos en pudrideros, tal como se ha apuntado también para otros yacimientos y horizontes temporales (Gómez et al., 2011; Esparza et al. 2012).

No obstante, la relativa abundancia de restos óseos craneales y mandibulares, coincidentes con su ausencia en algunas de las tumbas hace suponer, entre otros indicios, la posibilidad de que pudo haber en muchos casos una selección de determinadas porciones anatómicas, muy especialmente los cráneos y las mandíbulas para su posterior incorporación al mundo de los vivos como una parte noble e identitaria del difunto. Se trata de una práctica de amplísima tradición que arranca del Paleolítico y tiene un especial reflejo en las manifestaciones simbólicas del Neolítico del Próximo Oriente como ha puesto de relieve nuestra homenajeada (Rubio, 2004a).

\subsection{Mandíbulas humanas en el área central de Camino de las Yeseras}

Como muestra de la presencia de restos óseos del cráneo en distintos contextos no funerarios proponemos el ejemplo de las mandíbulas, fácilmente identificables y de conservación generalmente buena por lo que pudieron ser objeto de selección por parte de quienes trasladaron restos esqueléticos de sus allegados o ancestros fuera de los depósitos primarios de los cuerpos (ver fig. 2).

Diez de las hasta ahora dieciséis mandíbulas recuperadas en Camino de las Yeseras proceden de cinco unidades estratigráficas distintas del área central (tabla 2) (ver fig. 1). Se trata de un espacio con una superficie aproximada de unos $600 \mathrm{~m}^{2}$ en torno al cual se trazan cinco recintos de foso concéntricos. Es una gran estructura excavada, de base ligeramente cóncava, de tendencia rectangular y perfil lobulado debido a la existencia de diversos hoyos anexados. Está colmatada con un sedimento oscuro en el que se pueden apreciar 13 niveles horizontales y hasta 30 unidades de relleno con una profundidad máxima en la zona central de casi dos metros que indican que este relleno se ha producido en un espacio de tiempo prolongado y como consecuencia de una reiterada utilización de la zona (Ríos, 2011; Ríos et al., 2014).

La gran superficie y la potencia estratigráfica de esta amplia estructura unidas a la abundante cantidad de material entregado, entre el que destaca el importante volumen de restos faunísticos del que hasta la fecha disponemos de un estudio puntual, nos permite suponer que fue una zona de ocupación intensa y prolongada (Chorro, 2012). Posiblemente se trata de un área para diferentes usos, muchos de carácter colectivo, entre ellos el procesado y 


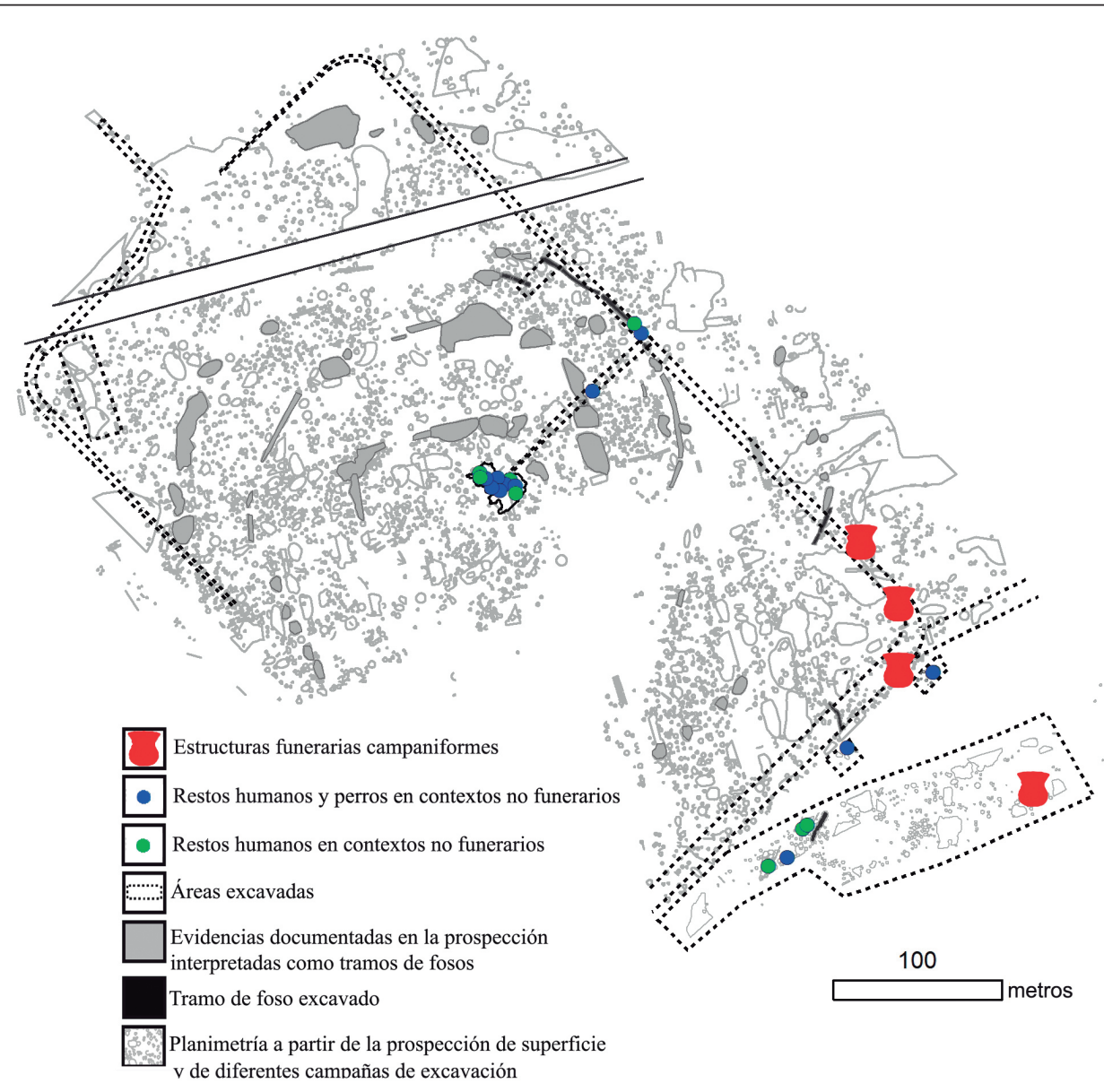

Figura 1. Planimetría de Camino de las Yeseras con distribución de los contextos funerarios campaniformes citados en el texto y de los contextos no funerarios con restos humanos (Argea Consultores S.L.-UAM).

muy probable consumo en este lugar de grandes mamíferos, como el uro, el vacuno, el caballo y el ciervo (Liesau, $2011 ; 2017)$. Ciertos indicios nos sugieren la permanencia de algunos huesos humanos en superficie durante algún tiempo, como indican huellas de mordeduras en varios de ellos, probablemente ocasionadas por carnívoros como los perros (Gómez et al., 2011: 118). Si bien la acumulación de mandíbulas y, en particular, las tres completas, podrían ser consecuencia de otras manipulaciones posteriores como su tratamiento y custodia en este lugar.

\subsection{Restos humanos en los fosos}

Muy significativo resulta también el hallazgo de uno de estos restos de un maxilar en un tramo del cuarto foso, todavía pendiente de estudio. Así mismo, otros fragmentos de frontal y dos húmeros han aparecido en el tramo norte de la entrada NE del cuarto foso de Camino de las Yeseras (ver fig. 1 y tabla 2). Este dato no tendría mayor interés si no estuviera en un contexto de nivel fundacional en la misma base del foso y asociado al depósito de un can completo y varias mandíbulas de otros individuos (fig. 3) (Liesau et al., 2013-14).

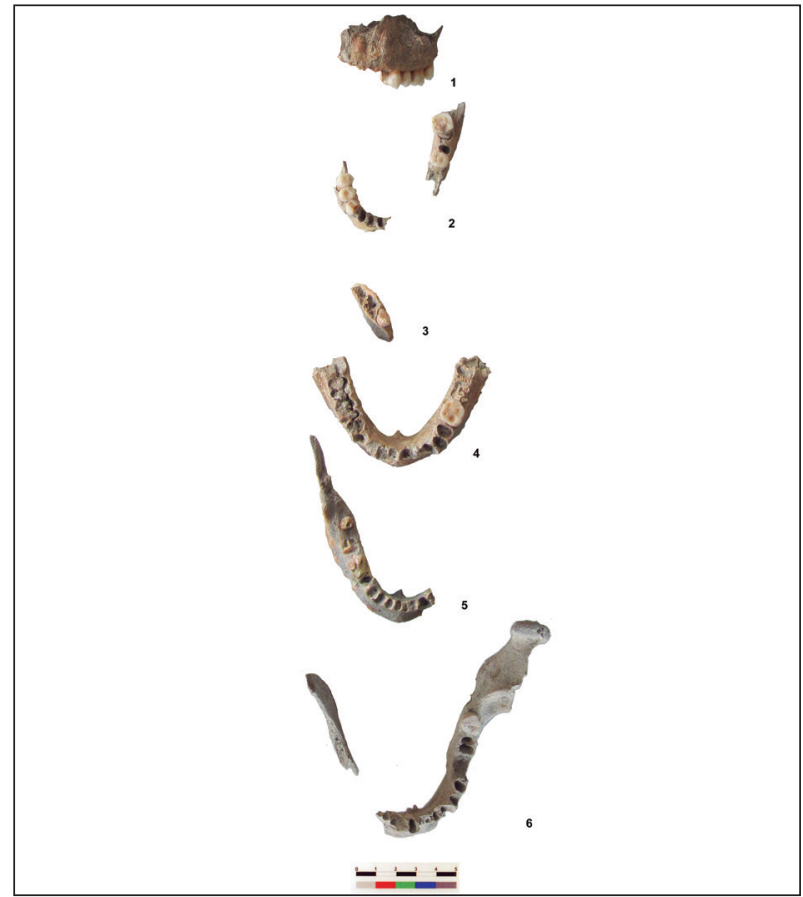

Figura 2. Selección de maxilar y mandibulas humanas procedentes de Camino de las Yeseras (maxilar, vista frontal; mandibulas, vista superior o craneal). 


\begin{tabular}{|c|c|c|c|c|c|}
\hline CONTEXTO & $\mathbf{N}^{\mathbf{o}}$ & $\begin{array}{c}\text { Ref. } \\
\text { excavación }\end{array}$ & $\begin{array}{l}\text { Mandíbulas humanas y/o } \\
\text { restos craneales }\end{array}$ & otros restos humanos & $\begin{array}{l}\text { Mandíbulas + otros restos } \\
\text { de perros }\end{array}$ \\
\hline \multirow{6}{*}{$\begin{array}{l}\text { Estructura tipo } \\
\text { CABAÑA }\end{array}$} & 1 & $\begin{array}{c}\text { F-322 } \\
\text { UE04/UE02 }\end{array}$ & $\begin{array}{l}\text { Mandíbula completa de } \\
\text { adulto }\end{array}$ & - & $\begin{array}{l}1 \text { hemimandíbula }+ \\
\text { otros restos } \mathrm{NMI}=1\end{array}$ \\
\hline & 2 & $\begin{array}{l}\text { F-411 } \\
\text { UE06 }\end{array}$ & $\begin{array}{l}\text { Fragmentos de calota craneal } \\
\quad+\text { otros restos } \mathrm{NMI}=1\end{array}$ & sí & - \\
\hline & 3 & $\begin{array}{c}\text { F-305 } \\
\text { UE05/UE12 }\end{array}$ & Restos craneales NMI $=1$ & - & \\
\hline & 4 & $\begin{array}{l}\text { A-125-126 } \\
\text { El. } 04 \text { UE01/ } \\
\text { UE02 }\end{array}$ & $\begin{array}{l}\text { Fragmento de mandíbula }+ \\
\text { restos calota craneal NMI=1 }\end{array}$ & & $\begin{array}{l}2 \text { hemimandíbulas } \\
\mathrm{NMI}=2\end{array}$ \\
\hline & 5 & $\begin{array}{l}\text { Corte Este } \\
\text { Estructura A }\end{array}$ & 1 mandíbula $\mathrm{NMI}=1$ & $\begin{array}{c}\text { Sí, tibia peroné y varios } \\
\text { huesos del pie }\end{array}$ & $\begin{array}{l}\text { Fragmento de hemimandíbula } \\
\quad+\text { otros restos } \mathrm{NMI}=1\end{array}$ \\
\hline & 6 & $\begin{array}{l}\text { Corte Oeste } \\
\text { Estructura A }\end{array}$ & Restos calota craneal & - & $\begin{array}{l}1 \text { Cráneo casi completo } \\
\mathrm{NMI}=1\end{array}$ \\
\hline $\begin{array}{l}\text { Hoyo sobre } \\
\text { cabaña } 305\end{array}$ & 7 & $\begin{array}{l}\text { F-438 } \\
\text { UE } 01\end{array}$ & $\begin{array}{l}\text { Fragmento de mandíbula con } \\
\text { incisivo }+ \text { otros restos } \mathrm{NMI}=1\end{array}$ & sí & - \\
\hline \multirow[t]{2}{*}{$\begin{array}{c}\text { Tramo del FOSO } \\
\text { IV }\end{array}$} & 8 & $\begin{array}{l}\text { ÁREA } 54 \\
\text { El A121 E01-A } \\
\text { UE } 01 / 02\end{array}$ & $\begin{array}{c}2 \text { frontales } \\
\mathrm{NMI}=2\end{array}$ & sí, dos húmeros & $\begin{array}{c}1 \text { perro completo }+ \\
3 \text { hemimandíbulas } \\
\text { posiblemente una de zorro } \\
\text { NMI }=4\end{array}$ \\
\hline & 9 & $\begin{array}{c}\text { ÁREA } 56 \\
\text { El 03-UE03 }\end{array}$ & $\begin{array}{l}\text { Fragmento de maxilar de } \\
\text { adulto }\end{array}$ & - & - \\
\hline \multirow{10}{*}{ ÁREA CENTRAL } & 10 & $\begin{array}{c}\text { A-132-I } \\
\text { El } 02 \text { UE101 }\end{array}$ & Mandíbula de adulto & sí, huesos largos & $\begin{array}{c}1 \text { maxilar }+2 \\
\text { hemimandíbulas }+ \text { otros } \\
\text { restos } \\
\mathrm{NMI}=3\end{array}$ \\
\hline & 11 & $\begin{array}{c}\text { A-132-I } \\
\text { El } 02 \text { UE104 }\end{array}$ & Mandíbula de adulto & sí, huesos largos & - \\
\hline & 12 & $\begin{array}{l}\text { A-132-I } \\
\text { El } 02 \text { U } 106\end{array}$ & $\begin{array}{l}\text { Fragmento de mandíbula de } \\
\text { adulto }\end{array}$ & sí, huesos largos & $\begin{array}{l}1 \text { canino } \\
\mathrm{NMI}=1\end{array}$ \\
\hline & 13 & $\begin{array}{c}\text { A-132-II } \\
\text { El } 02 \text { UE214 }\end{array}$ & $\begin{array}{l}\text { Fragmento de mandíbula de } \\
\text { adulto }\end{array}$ & - & $\begin{array}{l}1 \text { canino } \\
\mathrm{NMI}=1\end{array}$ \\
\hline & 14 & $\begin{array}{c}\text { A-132-II } \\
\text { El } 02 \text { UE204 }\end{array}$ & Fragmento de maxilar & - & - \\
\hline & 15 & $\begin{array}{l}\text { A-132-IV } \\
\text { El } 02 \text { UE404 }\end{array}$ & $\begin{array}{l}\text { Fragmento de maxilar y } \\
\text { mandíbula de maduro-senil }+ \\
\text { restos craneales NMI=1 }\end{array}$ & - & $\begin{array}{c}1 \text { mandíbula completa }+ \text { otros } \\
\text { restos } \mathrm{NMI}=1\end{array}$ \\
\hline & 16 & $\begin{array}{l}\text { A-132-IV } \\
\text { El } 02 \text { UE406 }\end{array}$ & $\begin{array}{l}\text { Fragmento de maxilar }+ \\
\text { restos craneales }\end{array}$ & - & - \\
\hline & 17 & $\begin{array}{l}\text { A-132-V } \\
\text { EL04 UEO1 }\end{array}$ & 1 maxilar & - & - \\
\hline & 18 & $\begin{array}{l}\text { A-132-V } \\
\text { UE } 504 \text { B }\end{array}$ & $\begin{array}{l}1 \text { fragmento de mandíbula de } \\
\text { adulto }\end{array}$ & - & $\begin{array}{c}1 \text { fragmento de } \\
\text { hemimandíbula }+ \text { otros restos } \\
\text { NMI }=1\end{array}$ \\
\hline & 19 & $\begin{array}{l}\text { A-132-V } \\
\text { UE } 506\end{array}$ & 1 mandíbula adulto & sí, 1 húmero & $\begin{array}{l}\text { Fragmentos de } 3 \text { maxilares } \\
\text { NMI }=3\end{array}$ \\
\hline
\end{tabular}

Tabla 2. Relación de algunos de los contextos no funerarios en los que se han documentado restos humanos (mandibulas, restos craneales y otros) asociados con restos de perro (mandibulas y otros) en Camino de las Yeseras. 


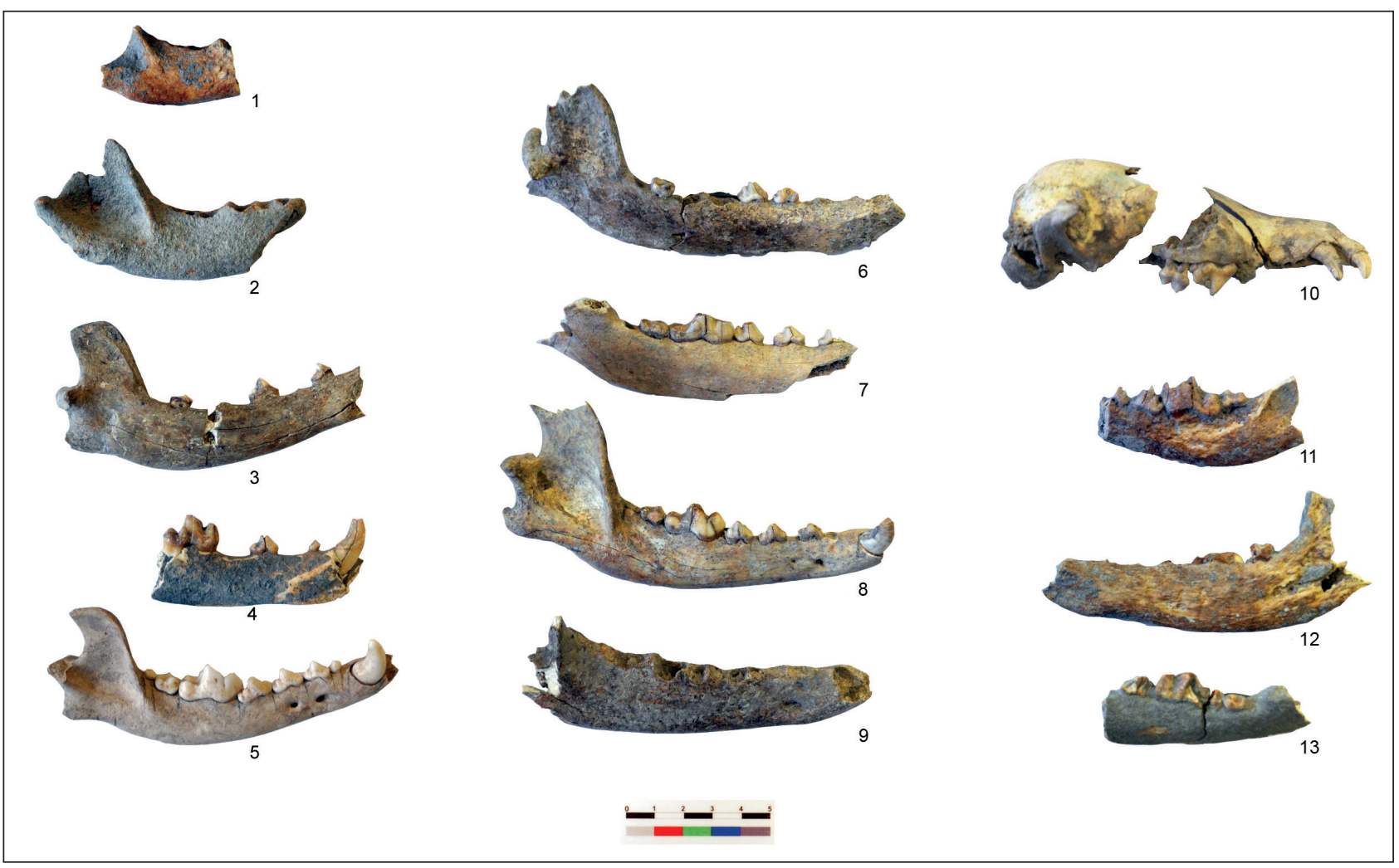

Figura 3. Selección de cráneo y mandíbulas de perro de fosos (1-5); área central (6-9) y cabañas (10-13) procedentes de Camino de las Yeseras (cráneo, vista lateral; mandibulas vista lingual y lateral).

\subsection{Restos humanos en estructuras de tipo cabaña}

Queda, por último, mencionar la presencia de sendas mandíbulas completas, en seis cabañas, a las que hay que sumar algún que otro maxilar o restos de calota craneal en estructuras asociadas como los hoyos abiertos en el interior de las mismas. El hecho de que varias piezas estén completas podría ser un indicio de que estamos ante un proceso de selección y de trasladados con una finalidad predefinida. Una sospecha que queda avalada también por los contextos a los que se asocian. En el sur del yacimiento nos interesa destacar la presencia de una mandíbula completa en el nivel de sellado de la cabaña 322, una estructura de especial significado si atendemos a su contenido, tanto por la abundancia de cerámicas campaniformes y no campaniformes decoradas, como por el volumen de restos faunísticos y la proporción que alcanza el porcentaje de especies silvestres entre las que destaca el uro y de nuevo, una mandíbula de perro, entre otros (Blasco et al. 2007).

La singularidad de este material nos permite suponer que la función de la "cabaña" no debió de ser estrictamente habitacional ya que en ella se pudieron desarrollar acciones de carácter productivo (talla lítica, industria ósea) pero también de índole simbólico, algunas de ellas quizás vinculadas a la memoria de los ancestros, lo que explicaría la presencia de dicha mandíbula junto con algunas "reliquias cerámicas" como es un lote de fragmentos campaniformes, de pequeño tamaño y bastante rodados, muy escasos en otros contextos supuestamente de uso habitacional (Ríos, 2011; Liesau et al., 2013).

La cabaña F-411 es otra gran cubeta con numerosas estructuras anexas y superpuestas y de prolongado uso. En ella se han documentado fragmentos de calota craneal humana en distintos niveles. En la cercana cabaña F-305 y dentro de un hoyo abierto en su interior (F-438) se recuperó un fragmento de mandíbula, cuyo contexto está todavía pendiente de estudio.

En el caso de la cabaña A del corte E con un contexto poco significativo en relación al uso, destacamos la recuperación de otra mandíbula sobre la base y en el centro de la estructura, una colocación que no parece casual, dada su asociación a un miembro inferior humano, un fragmento de cerámica campaniforme y nuevamente restos de un perro, entre los que destaca una mandíbula. Este hallazgo podría estar relacionado con un acto de clausura o incluso de amortización tras su abandono, aunque tampoco descartamos un posible rito fundacional de carácter propiciatorio. En la cabaña cercana, estructura A del corte Oeste, también podemos avanzar la recuperación de otros restos craneales humanos asociados a otro cráneo de perro (ver fig. 3 y tabla 2).

Por último, una mandíbula humana procedente del área 125-126, procede de un suelo de ocupación entre la puerta NE y la zona central. Aún pendiente de un estudio más exhaustivo, pensamos que al tratarse de una zona de paso hacia el área central puede ser un indicio con un significado muy especial, además de estar asociada, entre otros restos, a dos mandíbulas de perro (ver fig. 3 y tabla 2). 


\section{El trasiego de huesos humanos y otros ARTEFACTOS}

Pese a la escasez de paralelos publicados, la sustracción y recolocación de los restos humanos debieron de ser fenómenos frecuentes en las costumbres funerarias de estas sociedades, aunque no siempre hayan sido valoradas en comparación con la atención que han merecido los contextos primarios e intactos o simplemente no han sido reconocidos como tales, por tratarse de conjuntos mal conservados, estar mezclados con restos de fauna y sobre todo, por la dificultad de poder relacionarlos entre sí dentro de una dinámica de ocupación de larga duración.

Se trata de un fenómeno que afecta a tumbas colectivas en las que las manipulaciones posteriores a la esqueletización de los cuerpos adquieren cierta complejidad por la introducción de restos en un ámbito ya ocupado previamente por lo que requería una liberación de espacio para una nueva inhumación. Parece que esta reocupación y manipulación debió de ser más frecuente en las tumbas pertenecientes a personajes campaniformes que pudieron haber tenido una mayor relevancia entre la comunidad, o también para mantener unidos a individuos pertenecientes a una estirpe común o dominante. Aunque entre la población vecina sin ajuares campaniformes encontramos algún enterramiento secundario, no parece que esta práctica de traslados de restos haya sido ni tan frecuente ni tan compleja como entre los campaniformes. Ello induce a pensar que una buena parte de los restos humanos y parte de su ajuar, hallados fuera de las tumbas, debieron formar parte de ritos específicos post mortem, cobrando un gran significado la fragmentación de vasos cerámicos y extracción parcial de huesos en calidad de representar el tradicional concepto del pars pro toto.

Además, tenemos otros indicios de la relación campaniforme, como es la presencia de fragmentos decorados asociados a los restos humanos en tres de las cabañas analizadas (322, 305 y A del corte Este), así como asociaciones cronológicas que sitúan muchos de estos contextos dentro del intervalo temporal campaniforme entre el 2500 y el 1740 cal a.C. (Ríos, 2011, 2013).

Todo parece indicar que estos hábitos no son fruto de la improvisación, sino más bien consecuencia de unas costumbres arraigadas y previsibles que condicionaron la práctica de excavar las tumbas en los zócalos de estructuras amplias para darles un acceso lateral y no cenital como es habitual en otros cementerios campaniformes. De esta forma, se facilitaba un mejor acceso para el tránsito o la manipulación del contenido de las tumbas sin afectar la integridad del depósito, tanto para seleccionar lo que se deseaba extraer como, simplemente para poder visualizarlo. Esta posibilidad explicaría a su vez, por qué en otros conjuntos funerarios campaniformes con tumbas en hoyo simple, este tipo de fenómeno sea más puntual. En cambio, sí es más habitual en otras necrópolis de nuestra misma región geográfica, como es el caso de Huecas, donde las cuevas abiertas en ladera con acceso lateral a las cámaras, facilitan esta práctica de inhumaciones múl- tiples, reducciones y sustracciones (Barroso et al., 2015; Bueno et al. 2005, 2007-2008).

En Camino de las Yeseras parece que todas estas modalidades de sustracción y recolocación adquieren una gran importancia, aspecto que refuerza aún más el papel de los ancestros en la vida cotidiana del poblado, donde aparecen junto a estructuras integradas tanto en el área doméstica, como en espacios dedicados a alojar varios enterramientos, denominados Áreas Funerarias. La excepción sería la tumba "en fosa" del área 21, aunque también en este caso se abrió una pequeña cámara o covacha en la pared del gran hoyo en el que se encuentra, desde donde se podía observar y manipular su contenido. En consecuencia pensamos que estas prácticas parecen estar sujetas a unos determinados tipos de tumba y a su sistema de cierre, ya que a pesar de que algunos fueran de difícil apertura y de gran inversión de trabajo tanto en su construcción inicial como para su sellado, permitían la apertura y acceso a inhumaciones sucesivas y posteriores extracciones por no haber sido colmatadas las cámaras funerarias (Áreas Funerarias 1 y 2). No todas las tumbas fueron manipuladas, especialmente las que se clausuraron colmatando el espacio funerario con sedimento (A-36VII) o que sufrieron el colapso de su techumbre, sellando su contenido (A-36-X).

Uno de los aspectos más interesantes se ha podido investigar en el Área Funeraria 3, que inicialmente se denominó cabaña 5 por su semejanzas morfológicas y estructurales con los espacios domésticos (Blasco et al., 2005). Las características del registro cerámico y faunístico expresan un marcado carácter simbólico y delimitador espacial para recuperar pero también para exhibir determinados restos óseos encima de las tumbas como una auténtica expresión de la memoria de sus ancestros y de sus linajes, además de los bienes exóticos que la parafernalia funeraria demanda (Liesau et al. 2013). Este es un resultado poco reconocido y descrito hasta la fecha, en las que determinadas porciones esqueléticas se exhiben en los niveles de cierre o de sellado de tumbas y en las áreas funerarias.

En este sentido, tampoco es de extrañar que la elección estratégica del cinabrio en sus rituales funerarios sea una constante, no sólo en su vertiente simbólica alusiva a la sangre y a la vida por su llamativo color, sino por la necesidad de conservar sus cuerpos y huesos en las mejores condiciones posibles para su posterior recuperación, traslado y/o exhibición (Delibes, 2000; Liesau y Blasco, 2011-2012; Liesau, 2016)

En relación con los restos humanos en los fosos, para Camino de las Yeseras tenemos que destacar su presencia en contextos concretos, donde no aparecen mezclados con desechos de consumo, ni materiales cerámicos y líticos amortizados. Están por tanto en consonancia con los registros conocidos en otros yacimientos peninsulares donde se han localizado enterramientos parciales humanos, caso de Marroquíes Bajos, Valencina de la Concepción, La Pijotilla o Perdigões (Zafra et al., 2003; Hurtado, 2003; Fernández Gómez, 2013; Valera, 2010). Un panorama similar 
se ha reconocido en recintos europeos desde el Neolítico donde con frecuencia se localizan no sólo restos parciales y completos asociados a depósitos animales fruto de rituales muy distintos o como consecuencia de episodios violentos, actos de canibalismo, manipulaciones después del descarnado, e incluso actos simbólicos de carácter social (Whittle, 1996; Márquez y Jiménez, 2004; 2010; ZeebLanz et al., 2013; Valera y Silva, 2011; Valera, 2014).

En relación con la presencia de elementos craneales y mandíbulas humanas en estructuras de tipo cabaña, su ubicación en la base de la mayoría de ellas, podría tener un evidente significado simbólico fundacional de la estructura. No parecen responder a simples hallazgos casuales, pues su recuperación parcial o completa acompañada por mandíbulas de canes, formando parte del mismo contexto, tal vez indique que actuaban a modo de reliquia junto a los fragmentos de cerámica campaniforme o que fueron rápidamente enterrados en la base de estas estructuras con fines propiciatorios, afectivos, custodia de la memoria de los ancestros (tabla 2). Dada la estrecha relación afectiva y de guarda con las comunidades humanas, los perros adquieren especial protagonismo, también en la esfera simbólica, aspecto que también se conoce en otros ámbitos europeos, donde forman parte de actos de fundación y de clausura del ciclo vital o de uso e algunas estructuras (Bradley, 2005; Lazăr et al., 2016).

Pero la existencia de restos humanos en simples hoyos también merece ser discutida con más detalle, como es el depósito ritual de Calzadilla (Almenara de Adaja, Valladolid) que demuestra esa preocupación y definitiva amortización de determinados bienes de los ancestros en un hoyo de modestas dimensiones donde se depositan numerosos recipientes campaniformes, previamente fragmentadas o destacando las decoraciones simbólicas, junto a tan solo dos costillas humanas y desconcertantes asociaciones faunísticas en las que no falta el consumo o derramamiento de bebidas alcohólicas (Delibes y Guerra, 2004; Delibes y Herrán, 2007; Liesau et al., 2014).

Quedan por definir y estudiar mejor muchos casos de los comentados, como es el hecho de que todos los restos analizados en estos contextos pertenezcan a fundamentalmente a individuos adultos. Se hace necesario el estudio integral de estos complejos contextos, donde desde los artefactos a los depósitos faunísticos representan depósitos estructurados. En ellos también intervienen multitud de especies domésticas y silvestres, esqueletos completos o parciales y donde determinadas porciones animales marcan unas pautas deposicionales bien definidas. Tal sería el caso de los depósitos de perros y de otros cánidos con especial énfasis en sus mandíbulas, así como algunos restos craneales de bovinos (Liesau, 2012, Daza 2015). En Camino de las Yeseras, la asociación de mandíbulas humanas con otras de canes no es un hecho aislado, como así se atestigua en diferentes contextos (tabla 2). Pendiente de un estudio más exhaustivo ésta es una asociación que queremos destacar por encima de otras en relación con los hallazgos humanos en contexto no funerarios y que muy probablemente aluden al concepto del pars pro toto en el ideario simbólico, posiblemente de índole animista, de estas comunidades calcolíticas.

En definitiva, esta presencia de restos humanos fuera de las tumbas nos invita a enfocar el estudio de los contextos funerarios, por muy atrayentes que sean, dentro del contexto general del sitio en el que se encuentran, ya que la evidencia de la apertura de las tumbas supone la existencia de unas relaciones entre vivos y difuntos más allá de la muerte y nos obliga a buscar explicaciones del por qué y el para qué de unos expolios posiblemente consentidos, dentro de la complejidad de las prácticas simbólicas en las que también intervienen las mismas porciones anatómicas de animales que implican un tratamiento post mortem pautado del ideario de las comunidades de la Prehistoria reciente, donde los orígenes se remontan a periodos anteriores y dan continuidad a tradiciones posteriores.

\section{Bibliografía}

Andrés, T. (1998): Colectivismo funerario neo-eneolitico. Aproximación metodológica sobre datos de la cuenca alta y media del Ebro. Institución Fernando el Católico. Zaragoza.

Arteaga, C. (2011): "Sedimentología aplicada al estudio de una tumba del Área Funeraria 2: caracterización antrópica o natural". En C. Blasco, C. Liesau y P. Ríos (eds.): Yacimientos Calcolíticos con campaniforme de la Región de Madrid: Nuevos estudios. Patrimonio Arqueológico de Madrid, 6. Universidad Autónoma de Madrid. Madrid: 161-164.

Barroso, R.; Bueno, P.; Vázquez, A.P.; Odriozola, C.; Uribelarrea, D.; López, O.; de Balbín, R.; Peña, L. y Yravedra, J. (2015): “Campaniforme no funerario en la provincia de Toledoel yacimiento de Las Vegas. De nuevo el Valle de Huecas". Trabajos de Prehistoria, 72 (1): 145-157. DOI: https://doi.org/10.3989/ tp. 2015.12148

Blanco González, A. (2014): “¿Rutinas caseras o fiestas comunitarias? Tafonomía y remontaje de la cerámica calcolítica de El Ventorro (Madrid)". Complutum, 25 (1): 89-108. DOI: https://doi.org/10.5209/rev_ CMPL.2014.v25.n1.45357

Blasco, C.; Liesau, C.; Delibes, G.; Baquedano, E. y Rodríguez Cifuentes, M. (2005): “Enterramientos campaniformes en ambiente doméstico: El yacimiento de Camino de las Yeseras (San Fernando de Henares, Madrid)". En M. Rojo, R. Garrido e I. García (coords.): El Campaniforme en la Península Ibérica y su contexto europeo. Universidad de Valladolid: 457-479.

Blasco, C.; Delibes, G.; Baena, F.J.; Liesau, C. y Ríos, P. (2007): "El poblado calcolítico de Camino de las Yeseras (San Fernando de Henares, Madrid): Un escenario favorable para el estudio de la incidencia campaniforme en el interior peninsular". Trabajos de Prehistoria, 61 (1): 151-163.

Blasco, C.; Liesau, C.; Ríos, P.; Blanco, J.F.; Aliaga, R.; Moreno, E. y Daza, A. (2009): "Kupferzeitliche 
Siedlungsbestattungen mit Glockenbecher-und Prestigebeigaben aus dem Grabenwerk von el Camino de las Yeseras (San Fernando de Henares, prov. Madrid). Untersuchungen zur Typologie des Grabritus und zu dessen sozialer Symbolik". Madrider Mitteilungen, 52: 40-70.

Blasco C.; Liesau, C., y P. Ríos (eds.) (2011): Yacimientos calcolíticos con campaniforme en la Región de Madrid: Nuevos estudios. Patrimonio Arqueológico de Madrid, 6. Universidad Autónoma de Madrid.

Blasco, C.; Liesau, C.; Ríos, P.; Gómez, J.L. y Flores, R. (2014): "Un enterramiento múltiple del yacimiento calcolítico de Humanejos (Parla, Madrid) desde una perspectiva tafonómica: Agrupando y reagrupando la familia". Cuadernos de Prehistoria y Arqueología de la Universidad Autónoma de Madrid, 40: 11-29.

Bradley, R. (2005): Ritual and domestic life in Prehistoric Europe. Routledge. Londres.

Brück, J. (2006): "Fragmentation, Personhood and the Social Construction of Technology in Middle and Late Bronze Age Britain". Cambridge Archaeological Journal, 16 (3): 297-315. DOI: https://doi.org/10.1017/ S0959774306000187

Bueno, P.; Barroso, R. y Balbín, R. de (2005): "Ritual campaniforme, ritual colectivo. La necrópolis de cuevas artificiales de Valle de las Higueras (Huecas, Toledo)". Trabajos de Prehistoria, 62 (2): 67-90.

Bueno, P.; Barroso, R. y Balbín, R. de (2007-2008): "Campaniforme en las construcciones hipogeas del Megalitismo reciente al interior de la Península Ibérica". Veleia, 24-25: 771-790.

Chorro, $\mathrm{M}^{\mathrm{a}}$ de (2012): Estudio de la fauna calcolitica del Área central de Camino de las Yeseras (San Fernando de Henares, Madrid). Trabajo Fin de Máster. Departamento de Prehistoria y Arqueología. Universidad Autónoma de Madrid. (Inédito).

Daza, A. (2015): La fauna del Calcolitico de la región de Madrid: los depósitos de canes. UAM Ediciones. Madrid.

Delibes, G. (2000): “Cinabrio, huesos pintados en rojo y tumbas de ocre: ¿prácticas de embalsamiento en la Prehistoria?". En M. Olcina y J. Soler (coords.) Scripta in Honorem Enrique A. Llobregat Conesa. Alicante: 223-235.

Delibes, G. y Guerra, E. (2004): “Contexto y posible significado de un cuenco Ciempozuelos con decoración simbólica de ciervos hallado en Almenara de Adaja (Valladolid)". En E. Baquedano (ed.): Miscelánea en Homenaje a Emiliano Aguirre, Vol. IV: Arqueología. Museo Arqueológico Regional. Alcalá de Henares: 116-125.

Delibes, G. y Herrán, J.I. (2007): La Prehistoria. Biblioteca básica de Valladolid. Diputación de Valladolid. Valladolid.

Duday, H. y Guillon, M. (2006): “Understanding the circumstances of decomposition when the body is skeletonized". En A. Schmitt, E. Cunha and J. Pinheiro (eds.): Forensic Anthropology and Medicine: Com- plementary Sciences from recovery to cause of death. Humana Press Inc. Totowa, NJ: 117-158. DOI: https:// doi.org/10.1007/978-1-59745-099-7_6

Esparza, A.; Velasco, J. y Delibes, G. (2012): "Exposición de cadáveres en el yacimiento de Tordillos (Aldeaseca de la Frontera, Salamanca). Perspectiva bioarqieológica y posibles implicaciones para el estudio ritual funerario de Cogotas I". Zephyrus, 69: 95-128.

Fernández Gómez, F. (2013): “Las excavaciones del Museo Arqueológico de Sevilla en Valencina de la Concepción (Sevilla). En 1975-1976: Sectores de la Perrera, La Candeleda y Cerro de la Cabeza". En L. García Sanjuan, J.M. Vargas Jiménez, V. Hurtado Pérez, R. Cruz-Auñón Briones y T. Ruiz Moreno (eds.): El sentamiento prehistórico de Valencina de la Concepción (Sevilla): Investigación y tutela en el 150 aniversario del descubrimiento de La Pastora. Universidad de Sevilla, Sevilla: 131-150.

Gómez, J.L.; Blasco, C.; Trancho, G.; Grueso, I. y Martínez, M.S. (2011): "Los protagonistas". En C. Blasco, C. Liesau y P. Ríos (eds.): Yacimientos calcolíticos con campaniforme en la Región de Madrid: Nuevos estudios. Patrimonio Arqueológico de Madrid, 6. Universidad Autónoma de Madrid: 101-132.

Hurtado, V. (2003): "Fosos y fortificaciones entre el Guadiana y el Guadalquivir en el III milenio AC: evidencias del registro arqueológico". En S. Oliveira Jorge (coord.) Recintos murados da Pré-historia Recente, Porto/Coimbra, FLUP/CEAUCP: 141-268.

Kopytoff, I. (1986): "The cultural biography of things: commoditization as a process". En A. Appadurai (ed.): The social life of things: Commodities in cultural perspectives. Cambridge: 64-91. DOI: https://doi. org/10.1017/CBO9780511819582.004

Lazăr, C.; Mărgarit, M. y Bălăşescu, A. (2016): “Dogs, jaws, and other stories: Two symbolic objects made of dog mandibles from southeastern Europe". Journal of Field Archaeology, 41 (1): 101-117. DOI: https://doi. org/10.1080/00934690.2015.1114850

Liesau, C. (2011): "La Arqueozoología, un elemento clave en la concepción espacial de Camino de las Yeseras". En C. Blasco, C. Liesau y P. Ríos (eds.): Yacimientos calcolíticos con campaniforme en la Región de Madrid: Nuevos estudios. Patrimonio Arqueológico de Madrid, 6. Universidad Autónoma de Madrid: 167-170.

Liesau, C. (2012): "Depósitos con ofrendas de animales en yacimientos Cogotas I: antecedentes y características". En J.A. Rodríguez Marcos y J. Fernández Manzano (eds.): Cogotas I, Una cultura de la Edad del Bronce en la Península Ibérica. Homenajes a $M^{a}$ Dolores Fernández-Posse. Universidad de Valladolid, Consejería de Cultura y Turismo. Junta de Castilla y León: 219-257.

Liesau, C. (2016): "Some prestige goods as evidence of interregional interactions in the funerary practices of the Bell Beaker groups of Central Iberia”. En E. Guerra Doce y C. Liesau (eds.): Analysis of the Economic Foundations Supporting the Social Supremacy of the 
Beaker Groups. Proceedings of the XVII UISPP World Congress (1-7 September, Burgos, Spain). Oxford, Volume 6 / Session B36. Archaeopress Archaeology: 69-93.

Liesau, C. (2017): "Fauna in Living and Funerary Contexts of the 3rd Millennium BC in Central Iberia". En M. Bartelheim, P. Bueno Ramírez y M. Kunst (eds.): Key Resources and Sociocultural Developments in the Iberian Chalcolithic. Tübingen: 107-128.

Liesau, C.; Blasco.; Ríos, P.; Vega, J.; Menduiña, R.; Blanco, J.F.; Baena, J.; Herrera, T.; Petri, A. y Gómez, J.L. (2008): "Un espacio compartido por vivos y muertos: El poblado Calcolítico de fosos de Camino de las Yeseras (San Fernando de Henares, Madrid)". Complutum, 19 (1): 97-120.

Liesau, C. y Blasco, C. (2011-2012): "Materias primas y objetos de prestigio en ajuares funenarios como testimonios de redes de intercambio en el Horizonte campaniforme." Cuadernos de Prehistoria y Arqueología de la Universidad Autónoma de Madrid, 37-38: 209-222.

Liesau, C.; Ríos, P.; Aliaga, R.; Daza, A.; Llorente, L. y Blasco, C. (2013): "Hut structures from the Bell Beaker horizon: housing, communal or funerary use in the Camino de las Yeseras site (Madrid)". En P. Prieto y L. Salanova (coords.): Current researches on Bell Beakers Proceedings of the 15th International Bell Beaker Conference: From Atlantic to Ural. Poio (Pontevedra, Galicia, Spain): 139-151.

Liesau, C.; Vega, J.; Daza, A.; Ríos, P.; Menduiña, R. y Blasco, C. (2013-2014): "Manifestaciones simbólicas en el acceso Noreste del Recinto 4 del Foso en Camino de las Yeseras (San Fernando de Henares, Madrid)". Saldvie, 13-14: 53-69.

Liesau, C.; Guerra, E. y Delibes, G. (2014): “Casual or ritual: The Bell Beaker deposit of La Calzadilla (Valladolid, Spain)". Quaternary International, 330 (1): 88-96. DOI: https://doi.org/10.1016/j. quaint.2013.10.046

Liesau, C.; Ríos, P.; Vega, J.; Menduiña, R. y Blasco, C. (2014a): "Buscando los ancestros: La manipulación de los restos de las tumbas campaniforme en Camino de las Yeseras (San Fernando de Henares, Madrid)". Actas de las IX Jornadas de Patrimonio Arqueológico en la Comunidad de Madrid. Comunidad de Madrid: 137-148.

Liesau, C.; Vega, J.; Menduiña, R.; Daza, A.; Ríos, P. y Blasco, C. (2014b): "El simbolismo animal en áreas de tránsito de un recinto de fosos: el ejemplo de Camino de las Yeseras (San Fernando de Henares)". Actas de las X Jornadas de Patrimonio Arqueológico en la Comunidad de Madrid. Comunidad de Madrid: 191203.

Márquez, J.E. y Jiménez, V. (2004): “Muerte ubicua: Sobre deposiciones de esqueletos humanos en zanjas y pozos en la Prehistoria Reciente de Andalucía". Mainake, 26: 115-138

Márquez, J.E. y Jimémez, V. (2010): Recintos de fosos. Genealogía y significado de una tradición en la Prehis- toria del suroeste de la Península Ibérica (V-III milenios a.C.). Universidad de Málaga.

Márquez, J.E. y Mata, E. (2016): “¿Qué cosa es la llamada recinto de fosos? Una revisión crítica a un concepto arqueológico en formación”. Cuadernos de Prehistoria y Arqueología de la Universidad Autónoma de Madrid. Anejos 2: 39-48.

Marshall, Y.M. y Gosden, C. (eds.) (1999): "The Cultural Biography of Objects". World Archaeology, 32: 169178.

Ríos, P. (2011): "Nuevas fechas para el Calcolítico de la región de Madrid. Aproximación cronocultural a los primeros poblados estables". En C. Blasco, C. Liesau y P. Ríos (eds.): Yacimientos calcolíticos con campaniforme en la Región de Madrid: Nuevos estudios. Patrimonio Arqueológico de Madrid, 6. Universidad Autónoma de Madrid: 73-86.

Ríos, P. (2013): "New dating of Bell Beaker horizon in the region of Madrid". En P. Prieto Martinez y L. Salanova (eds.): From Atlantic to Ural: mobility and local evolution during the 3rd millennium BC in Europe: 97-109.

Ríos, P.; Liesau, C. y Blasco, C. (2014): "Funerary practices in the ditched enclosures of Camino de las Yeseras: Ritual, Temporal and Spatial Diversity". En A. Valera (ed.): Recent Prehistoric Enclosures and Funerary Practices in Europe. Proceedings of the International Meeting hed and the Gulbenkian Foundation. (Lisbon, Portugal, November 2012). BAR International Series 2676. Archaeopress, Oxford: 139-149.

Rubio, I. (2001-2002): “El mundo funerario neolítico peninsular: algunas reflexiones sobre su transfondo social". Anales de Prehistoria y Arqueología, 17-18: 53-66

Rubio, I. (2004a): "Rituales de cráneos y enterramiento en el neolítico precerámico del Próximo Oriente". Cuadernos de Prehistoria y Arqueología de la Universidad Autónoma de Madrid 30: 27-45.

Rubio I. (2004b): "Religión y procesos de cambio en el Neolítico precerámico del Próximo Oriente”. Isimu. Revista sobre Oriente Próximo y Egipto en la antigüedad, 7: 133-168.

Rubio, I. (2010-2011): “Cerámica y simbolismo: Posibles interpretaciones de algunas cerámicas peninsulares del Neolítico antiguo". Boletín de la Asociación Española de Amigos de la Arqueología, 46: 31-52

Valera, A. (2010): "Ossos humanos provenientes dos fossos 3 e 4 e gestão da norte nos Perdigões. Apontamentos de Arqueologia e Património, 6: 29-39.

Valera, A. y Silva, A.M. (2011): "Datações de radiocarbono para os Perdigões (1): contextos com restos humanos nos sectores I \& Q.” Apontamentos de Arqueologia e Património, 7: 7-14.

Valera, A. (ed.) (2014): Recent Prehistoric Enclosures and Funerary Practices in Europe. Proceedings of the International Meeting held at the Gulbenkian Foundation (Lisbon, Portugal, November 2012) British Archaeological Report BAR, International Series 2676. Archaeopress, Oxford. 
Vega, J.; Blasco, C.; Liesau, C.; Ríos, P.; Blanco, J.F.; Menduiña, R.; Aliaga, R.; Moreno, E.; Herrera, T.; Petri, A. y Gómez, J.L. (2010): "La singular dualidad de enterramientos en el poblado de silos Calcolítico de Camino de las Yeseras (San Fernando de Henares, Madrid)". En J. Fernández Eraso y J.A. Mujika (eds.): Actas del Congreso Internacional sobre Megalitismo y otras manifestaciones funerarias contemporáneas en su contexto social, económico y cultural. Munibe Suplemento, 32: 648-662.

Whittle, A. (1996): Europe in the Neolithic: The Creation of the New Worlds. Cambridge University Press. Cambridge.
Zafra de la Torre, N.; Castro López, M. y Hornos Mata, F. (2003): "Sucesión y simultaneidad en in gran asentamiento: la cronología de la macro-aldea de Marroquíes Bajos, Jaén. c. 2500-2000 cal ANE". Trabajos de Prehistoria, 60 (2): 79-90. DOI: https://doi.org/10.3989/ tp.2003.v60.i2.82

Zeeb-Lanz, A.; Haack, F. y Bauer, S. (2013): “Menschenopfer- Zerstörungsrituale mit Kanniblismus- Schädelkult: Die Aussergewöhnliche Bandkeramische Anlage von Herxheim (Südpfalz)". Mitteilungen des Historischen Vereins der Pfalz, 111: 5-53. 